\title{
Research on Modeling and Simulating of Harmonic Load Based on APF

\author{
Pu Xie ${ }^{1, a}$, Jin-cheng Zhao ${ }^{1, b}$ and Zheng-chun Liü, \\ ${ }^{1}$ Mechanical Engineering College, Shijiazhuang 050003, China
} \\ axhpjhc2007@163.com, bincheng62@126.com, ${ }^{\text {c }}$ nini82124@sina.com
}

Keywords: harmonic load; APF; three-phase four-wire system; MATLAB

\begin{abstract}
With the development of power electronic technology, harmonic load is one of the main reasons for damage to the power quality of the limited capacity power system. Firstly, the functional requirements of harmonic load model in the three-phase four-wire isolated power supply system are carried out according to the test data and waveform. And then, a general harmonic load model based on APF (Active Power Filter) is proposed, in which the odd order harmonic current can be afforded by specified value. In order to realize the accurate control of harmonic current, dual closed-loop control strategy based on the synchronous coordinate transformation is adopted. Finally, the simulation model is established and the control strategy of harmonic load model based on APF is varied by a large number of simulation tests.
\end{abstract}

\section{Introduction}

With the development of power electronic technology, harmonic load is one of the main reasons for damage to the power quality of the limited capacity power system[1].Harmonics is one of the power quality issues that influences to a great extent rotary machine vibration, voltage quality degradation and destruction of electric power components [2]. At present, most of the harmonic analysis of power system is studied on the grid, and the detailed analysis of the harmonic load is not carried out [3]. When it comes to the modeling and simulation of the harmonic load, specific equipment or circuit is constructed. Although the simulation model based on concrete circuit is accurate and clear, it is only for a specific equipment to carry out the harmonic analysis, whose general purpose is poor [4]. Therefore, this paper attempts to put aside the research ideas based on specific circuit structure. According to the basic principle of harmonic generation mechanism, a general harmonic load model based on APF is proposed, in which the odd order harmonic current can be afforded by specified value.

\section{Functional Requirement Analysis of Harmonic Load Model}

The load characteristics of the finite capacity power system are different from that of the grid power supply system. The power system is a three-phase and four-wise power supply system. In order to study the interaction effect between harmonic load and power supply, the model should have characters such as generality and adaptability. The general harmonic load model can simulate the bus harmonic current under various conditions.

From the test data of the specific $t$ instance, the following functional requirements are proposed for the model:

1. The limited capacity power system is always supplied by the diesel generating set, so the general harmonic load model should be adapted to three-phase four wire power supply system;

2. Most of the power system don't contain the even harmonic content, so only the odd harmonic content should be controlled, that is $3,5,7,9,11,13$ harmonic content is controllable. The highest harmonic content of the model is 13 because other harmonic content is very low in the power supply system.

3. The dynamic response of the model should be fast and the steady state accuracy is high, and the control algorithm should be simple to be realized by hardware easily. 


\section{Design Principle of Harmonic Load Model}

Based on the functional requirements analysis of the nonlinear harmonic load model, the general harmonic load is designed according to the principle of active power filter. As we all known, an active power filter is used to compensate harmonics current $[5,6]$. While in the general harmonic model, the APF is used to generate custom defined harmonic current. Although the two functions of APF are completely different, the harmonic generation mechanism is the same. So the current tracking and controlling methods of APF are worthy referencing.

The harmonic load model is consisted of two parts: one is the main circuit and the other is control system. The main circuit is a PWM converter that is made of power electronic devices (GTO or IGBT) in which capacitors are used as DC storage elements. The function of main circuit is to compensate or absorb the harmonic content of the bus, so that the number of harmonic current of bus current is the same with the specified value. Control system is included harmonic current given unit, command current calculating module, pulse control signal processing and driving module.

The fundamental ideas and principles of harmonic load model are as following:(1)Get the instruction signal by transforming and calculating the harmonic current specified and the bus current.(2)Generate pulse control signal by calculation.(3)Driven the main circuit and generate harmonic compensate current $i_{\text {cabc }}$ so that the number of harmonic current of bus current is the same with the specified value. On the basis of the analysis of APF control method, the principle of nonlinear harmonic load model is designed, as shown in figure1.

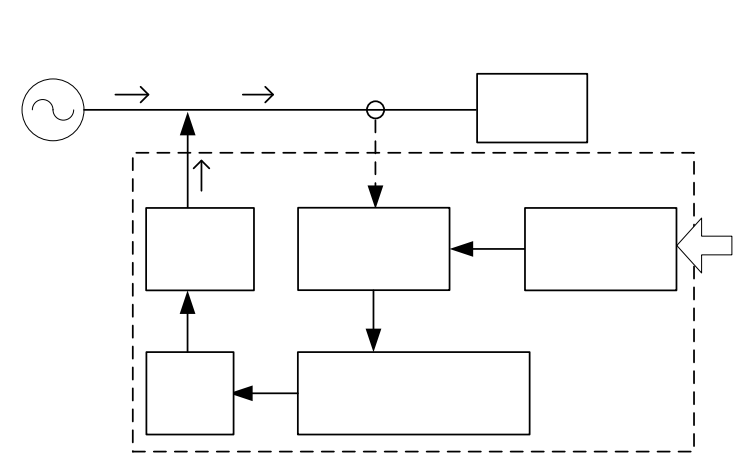

Fig.1 Principle diagram of model

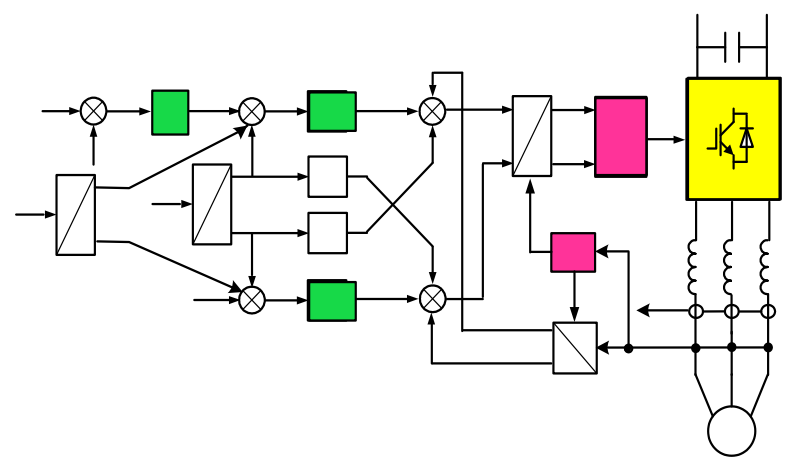

Fig.2 Design diagram of harmonic load model

\section{Control module design of nonlinear harmonic load based on MATLAB}

The control strategy for nonlinear harmonic load model In order to realize the accurate control of harmonic current, dual closed-loop control strategy based on the synchronous coordinate transformation is adopted, which is seen in Fig.2.

The voltage signal of the sensor module is collected and processed and then is converted into phase voltage. The signal of phase voltage is connected with locked loop and the phase voltage angle of the motor is calculated, as the vector angle of the coordination transformation. The fundamental active current is given by the PI controller in the voltage outer loop controller. The harmonic current given is added with the fundamental active current by the coordinate transformation and the voltage value of the two phase synchronous rotating coordinate system is obtained by the proportional integral resonant controller. Finally voltage value of the two phase synchronous rotating coordinate system is re-transformed and the pulse of IGBT is given by the space vector modulation method.

All the control of nonlinear harmonic load is based on the synchronous coordinate transformation, including the $i_{\text {sabc }}$ and the specified harmonic current $i^{*}$ habc. Both of them is first transformed by Park transformation and then the error is compared. The current-loop control and voltage feed-forward decoupling is based on the synchronous coordinate transformation. The simulation model based on Matlab/Simulink of three phase rectifier control is shown in fig.3 


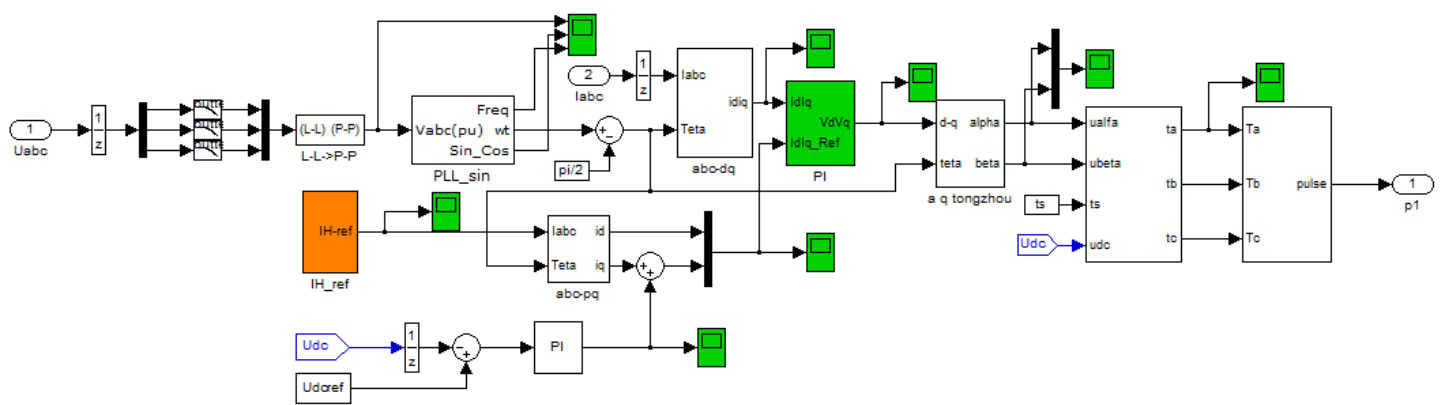

Fig. 3 ' I he simulation model of three pnase rectitier control

Phase locked loop For three phase rectifier control algorithm, the vector control need accuracy coordination transformation vector angel. So the speed of the controller and the accuracy of the input phase locked loop are very important. The angle of the phase voltage can be calculated according to the input of the three-phase voltage by Phase locked loop.

Harmonic current given The harmonic current given model is shown in figure 4. If the user specify the amplitude and times of the harmonic current then select and open" Flie->Model Properties->Call backs->InitFcn", which is seen in fig.4, the harmonic current is as following. "A5" is the 5 times harmonic current, whose amplitude is $5 \mathrm{~A}$," $\mathrm{k} 5$ ” means the harmonics times is 5 .

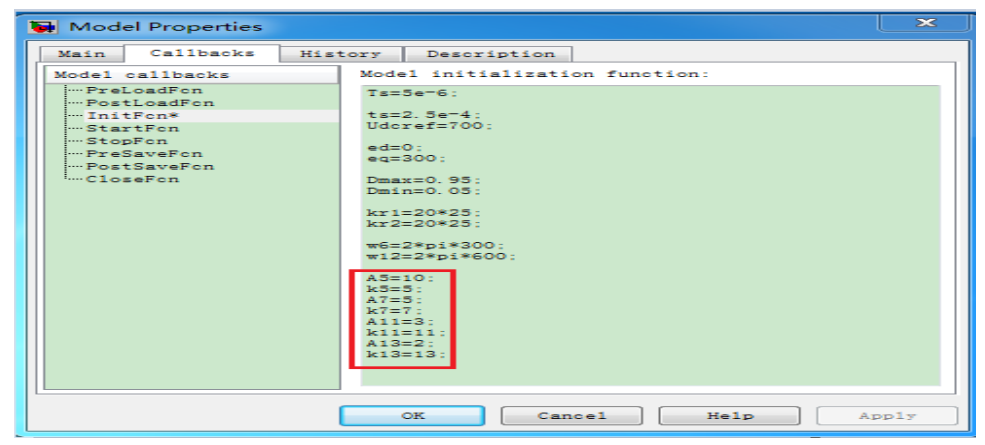

Fig.4 Harmonic current given model

Fig.5 System diagram of independent power supply and limited capacity power system model

The simulation model of whole system The simulation model of whole system is consisted of AC synchronous generator, rectifier bridge, capacitance of direct current side, resistance and other components. The power supply system is alternate current synchronous generator and alternate current side is connected with IGBT three-phase full controlled rectifier bridge. A given harmonic times and harmonic content is the same with Fig.4. The harmonics current content is $i_{5 \text { th }}=10 \mathrm{~A}$, $i_{7 \mathrm{th}}=5 \mathrm{~A}, i_{11 \mathrm{th}}=3 \mathrm{~A}$ and $i_{13 \mathrm{rd}}=2 \mathrm{~A}$, which achieved by the closed-loop control, whose simulation model is as in fig. 5 .

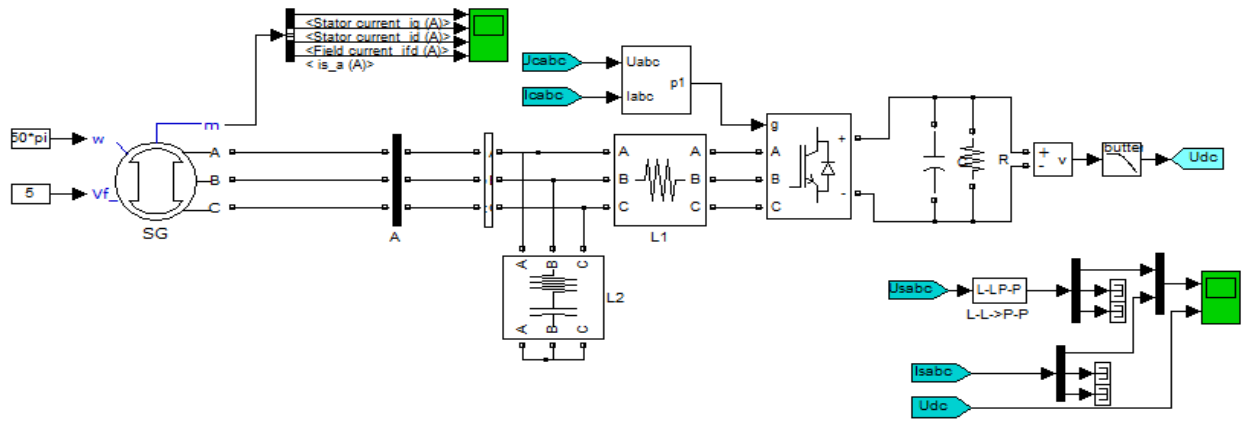

Fig.5 System diagram of independent power supply and limited capacity power system model

Synchronous motor rated capacity of the system is 60KVA, rated speed 1500rpm, rated frequency $50 \mathrm{~Hz}$, rated voltage $400 \mathrm{~V}$, DC side resistance is $49 \mathrm{ohm}$, DC side voltage is given as 
700V. Simulation results are shown in Figure 6, it can be seen that the feedback of DC voltage follower is given, stable at around $700 \mathrm{~V}$.

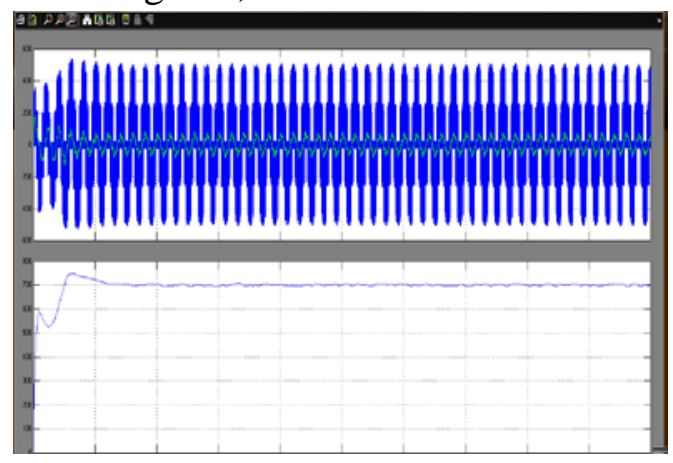

Fig.6 Phase voltage and current

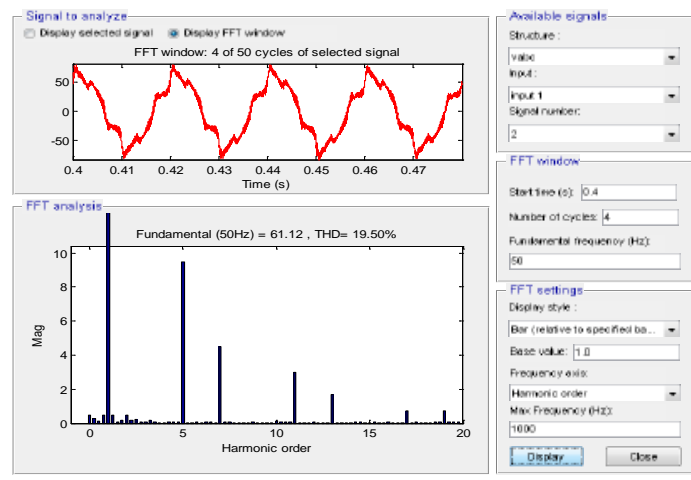

Fig.7 FFT analysis of phase current

The functional requirements of harmonic load model in the three-phase four -wire isolated power supply system are carried out according to the test data and waveform. And then, a general harmonic load model based on APF is proposed. According to a certain three-phase four -wire isolated power supply system, the power level of the system and the corresponding maximum current value are determined when the main circuit parameters are determined. So when the current value of the fundamental current and harmonic current is given, the total current value must be guaranteed not to exceed the maximum current value of the system.

\section{Acknowledgement}

This paper is supported by National Natural Science Foundation of China (No. 51307184).

\section{References}

[1] F.Liu,X.Zhang,Z.Xie,P.Xu,and L.Chang. Shunt active power filter for harmonic and reactive current compensation in wind conversion systems, in 2008 Proc. IEEE PESC Conf.,pp. 2329-2332.

[2] B.Singh,G.K.Kasal,A.Chandra,andK.AI-Haddad. Improved power quality based controller for a 3-phase 4-wire isolated wind energy system, in2007Proc.EPC Conf,pp.531-536.

[3] J.Mazumdar,R.G.Harley,F.C.Lambert,andG.K.Venayagamoorthy,Neural network based method for predicting nonlinear load harmonics. IEEE Trans. Power Electron., vol.22, no.3, pp.1036-1045, May2007.

[4] S.A.O.daSilva,S,A.F.Neto,S.G.S.Cervantes,A.Goedtel,and CFNascimento. Synchronous reference frame based controllers applied to shunt active power filters in three-phase four-wire systems, in ConfProc.IEEE ICIT,int.Conf. Industrial Technology, 2010, pp.832-837.

[5] Li Ma,Jingai Zhou, Zhengyu Lu. An improved harmonic detecting apporach for active power filter[J].Power Electronics and Motion Control Conference, 2000,3: pp.1420-1424.

[6] Chang G W. A new method for determing reference compensating current of three phase shunt active power filter[J]. IEEE Power Enginering Review.2001,21(3), pp.63-65. 